\title{
Erratum to: Inhibition of Notch signaling facilitates the differentiation of human-induced pluripotent stem cells into neural stem cells
}

\author{
Chun-Yuan Chen · Wei Liao • Yuan-Lei Lou • \\ Qing $\mathbf{L i} \cdot$ Bin $\mathrm{Hu} \cdot$ Yang Wang $\cdot$ Zhi-Feng Deng
}

Published online: 2 August 2014

(C) Springer Science+Business Media New York 2014

\section{Erratum to: Mol Cell Biochem}

DOI 10.1007/s11010-014-2130-3

The Graduate School of Nanchang University, the second corresponding authors' institution, is added to the corresponding authors' affiliations. The corrected version of the paper failed to be submitted to the editor office attributed to the bad network. Because this work is closely related to Dr. Chen's graduation from Nanchang University, the corrected affiliations are presented here.

Everything else in the paper remains correct.

The online version of the original article can be found under doi:10.1007/s11010-014-2130-3.

C.-Y. Chen · W. Liao - Y. Wang · Z.-F. Deng

Graduate School of Nanchang University, Nanchang 330006,

China

Y.-L. Lou

Institute of Urology, The First Affiliated Hospital of Nanchang

University, Nanchang 330006, China

Q. Li $\cdot$ B. Hu $\cdot$ Y. Wang $(\bowtie)$

Institute of Orthopaedic Surgery, Shanghai Jiaotong University Affiliated Sixth People's Hospital, Shanghai 200233, China

e-mail: wangy63cn@126.com

\section{Z.-F. Deng $(\bowtie)$}

Department of Neurosurgery, Shanghai Jiaotong University Affiliated Sixth People's Hospital, Shanghai 200233, China e-mail: dengzf63@126.com 